\title{
Global ageing: challenges and perspectives
}

\author{
V.A. Chereshnev ${ }^{1}$, V.H. Khavinson ${ }^{2}$, O.N. Mikhailova, ${ }^{2, *}$, I.G. Popovich ${ }^{2}$, \\ M.V. Chereshneva ${ }^{1,}$
}

\author{
${ }^{1}$ Institute of Immunology and Physiology, RAS Ural Branch, 620049, Pervomaiskaya st., 106, \\ Yekaterinburg, Russia \\ ${ }^{2}$ St. Petersburg Institute of Bioregulation and Gerontology, 197110, Dinamo pr., 3, St. Petersburg, \\ Russia
}

The evidence of the global population ageing elucidates major challenges and opportunities of the 21 st century. The most comprehensive document on ageing which determines the contents for international collaboration and provides guidelines and recommendations for building national capacity is the Madrid International Plan of Action on Ageing (MIPAA) adopted in 2002. MIPAA has recognized population ageing as a 'universal force that has the power to shape the future as much as globalization' and called on the international community to 'embrace the potential of the ageing population as a basis for future development' [1]. The 2017 UN Report features data on the increase in life expectancy during the recent decades [2]. On the global scale, life expectancy at birth has risen by 3.6 years during 2000-2015, i.e. from 67.2 to 70.8 years. According to UN forecasts, by 2030 the population of our planet aged 60 and over will amount to $3.1 \mathrm{bn}$ people. From 2000 up to 2050 , the number of people aged over 80 in the total population structure is predicted to increase from $70 \mathrm{mln}$ to $401 \mathrm{mln}$, respectively. Thus, a global demographic imbalance is getting worse. In 40 years period, the number of people over 60 will exceed that of people under 15 . Low birth rates and increased life expectancy in many countries are certain to cause problems in the labor market, and lack of labor resources in particular. Population ageing is one of the most important factors affecting politics in the multi-dimensional context of social and economic changes and transformations in the labor market. In this connection, the main reforms should be directed at using the potential of all generations and, particularly, that of the growing elder population.

It is important to understand that the solution to the problems concerned with population ageing is only possible if all society members are aware that the citizen aged 65 and older constitute important potential for social development [3,4]. As a rule, healthy people of older age are able to work till later age, compensating for the work force deficit caused by lower birth rate, thereby providing for the continuity of knowledge and experience of older generations. It is necessary to realize that the enhancement of the physiological resources of human vitality and healthy longevity cannot be implemented unless modern technologies are applied; programs for encouraging healthy life style are developed and implemented; educational and wellness strategies for persons of all ages are introduced; realistic approaches are implemented to financial support at the later stages in life; programs and plans concerned with ageing issues are coordinated.

The ageing process must be considered not only in terms of ethical and social problems; it should be studied as a special physiological mechanism that has a certain evolutionary

\footnotetext{
* Corresponding author: ibg@gerontology.ru
} 
function. Humans species specific limit is by $30-40 \%$ higher than the actual average life span. The influence of various unfavorable factors on human body leads to changes in the gene expression and structure, which are accompanied by protein synthesis disruption and reduction of organism functions. Ageing has many dysfunctional levels and can be classified as a systemic syndrome [5,6]. Ageing is characterized by the reduction of the body adaptability due to a decline in the functions of cells, systems and organs. Therefore, many researchers focus on the changes occurring in the major body systems: immune, cardio-vascular, central nervous and endocrine ones. The symptoms of ageing can be traced already at the end of the reproductive period and become more evident with further ageing. In the late 19th century, I. I. Mechnikov confirmed that improvement of cell immunity contributes to life span increase. He developed the phagocyte immunity theory in the belief that the human body has an inherent capacity to withstand pathological ageing (Nobel Prize in Physiology or Medicine shared with P. Erlich, 1908) [7]. A hundred years later, P. Doggerty and P. Zinkernagel accomplished detailed research of cell immunity specificity at viral infection, which largely confirmed Mechnikov's conclusions (Nobel Prize in Physiology or Medicine, 1996). In 1962, D. Watson and F. Crick received a Nobel Prize in Physiology or Medicine «for the discovery of the molecular structure of nucleic acids and its role in transferring information in live tissues». In 1961, F. Jakob and J. Monod proposed a genetic regulation model of protein synthesis involving low-molecular collagen, which displaces the depressor triggering allosteric conformational transition in the DNA structure and the bacterial cell (Nobel Prize in Physiology or Medicine shared with A.Lvov, 1965) [8].

As a result of a lengthy work, M. Nirenberg and H. Khorana managed to decipher the genetic code and determine the codons (nucleotide triplets) for each of the twenty amino acids (Nobel Prize in Physiology and Medicine shared with R. Holley, 1968). Fundamental research into the biochemistry of nucleic acids and determination of the RNA and DNA sequences were accomplished in the 60-ties and 70-ties of the 20th century by P. Berg, W. Gilbert and F. Sanger (Nobel Prize in Chemistry, 1980). All these fundamental discoveries in molecular biology formed the basis for research into theoretical and experimental gerontology. It should be emphasized that application of geroprotectors to physiologically stimulate an ageing body is scientifically based on modern concepts of ageing mechanisms, which means that we can virtually influence and, to a certain extent, control human ageing process $[9,10]$.

Searching for new effective means of influencing an ageing body, finding the most rational combinations of geroprotective agents is a vital task. During a recent decade the achievements in theoretical and applied gerontology allowed implementing targeted regulation of age-related changes. The progress in practical medicine is obviously dependent on the research achievements in molecular medicine, i.e. genetics, molecular and cell biology which often result in creation of new medications and technologies. By now, there have been identified genes which regulate the mechanisms of individual development and occurrence of many diseases [9].

As the cell proliferation and differentiation processes are reduced with age, there is a chance of correcting these dysfunctions by monitoring gene expression. Research into the genetic mechanisms of ageing and development of age-related pathologies forms the basis for regular therapy, involving application of transcription modulators, which hinder and reverse age-related genetic changes. Development of effective bioregulators facilitating achievement of species-related life span limit and preservation of the main physiological functions is one of the most urgent problems in modern biogerontology.

Of special interest is the restricted calorie diet (RCD). One of the most well-known studies is the work by L. Fontana et al. [11], which evaluated the reduction of calorie consumption in people who had been on this diet for 6 years on average, as compared to the 
healthy controls who had been on an ordinary diet. As a result it was established that the people who had been on a restricted calorie diet had lost weight, reduced the levels of general cholesterol, low-level lipoprotein, triglyceride, glucose, insulin, C-reactive protein, etc., as compared to the control group. These data suggests that RCD significantly reduces the risk of developing the metabolic syndrome and ischemic heart disease [12]. It was also established that the people who voluntarily restrict their food, and hence calorie consumption live longer than those who do not.

Recent investigations manifested a decrease in cardio-metabolic risk factors in humans without signs of obesity who followed moderate calorie restriction. These data point out potential advantages and usefulness of the moderate caloric restriction for cardio-vascular health of young and middle-aged people [13]. One of the mechanisms providing positive effect of the restricted calorie diet is increased body sensitivity to insulin, which prevents development of a number of age-related diseases and ageing.

One of the popular ageing theories is that of free radicals, proposed by D. Harman [14], who stated that over time, organisms accumulate macromolecular cell damage (of DNA, proteins and lipids), caused by free radicals, mainly by reactive oxygen forms. Accumulation of such damage causes cardio-vascular, neurodegenerative and oncological disorders, as well as age-related immunodepression and a number of other pathological changes. It is believed that reactive oxygen forms damage the membranes, collagen, DNA, chromatin, and structural proteins, and are also involved in epigenetic maintenance of nuclear and mitochondrial gene regulation, influencing the intracellular calcium level, etc. [15]. The fact that the products of reactive oxygen interaction with macromolecules persistently occur in various organs and tissues shows that the anti-oxidative systems are not effective enough and that the cells are subject to oxidative stress. Enhancement of antioxidative protection could play a substantial role in the geroprotective mechanism.

Among antioxidants, a special place belongs to resveratrol, which is a vegetable polyphenole [16]. In experimental research resveratrol was shown to increase the life span of yeast, worms and flies, as well as short-living fish [17]. Resveratrol also exhibit antitumor activity, and is considered a potential candidate for prevention and treatment of several types of cancer [18]. Moreover, other bioactive effects, namely as antiinflammatory, cardioprotective, vasorelaxant, phytoestrogenic and neuroprotective have also been reported. One of them stated that patients with type II diabetes who were receiving resveratrol (2x5 mg per day) showed significant improvement of insulin sensitivity, reduction of oxidative stress and improvement in the activation level of $\mathrm{B}$ protein kinase, as compared to the patients who were receiving placebo [19]. Geroprotective antioxidation mechanisms of resveratrol action are largely similar to those of the restricted calorie diet [20]. This is testified by numerous studies, which describe the ability of resveratrol to prevent the development of type II diabetes, as well as some neurodegenerative disorders (Altzheimer disease), lower the protein biosynthesis rate and gene expression, reduce oxidative stress and prevent the development of cardio vascular [21] and cancer diseases [22].

In recent decades much attention in world science has been paid to the physiological role of melatonin, especially in the ageing process. It has been established that in many ageing people the level of melatonin, the pineal indole hormone, is significantly decreased. Melatonin regulates the sleep-wake cycle, changes in body motion and temperature, and influences seasonal activity cycles as well as reproduction. Most of the experimental research proves melatonin to possess geroprotective properties, since it both increased the animals' longevity and slowed down the ageing of their reproductive system [23]. Melatonin has an anti-carcinogenic and anti-tumor effect, which has been demonstrated on the models of spontaneous and induced carcinogenesis in animals [24]. The geroprotective and anticarcinogenic effect of melatonin is due to its anti-oxidative activity [25]. There are 
reasons to believe that an important role in the geroprotective effect of melatonin is played by its normalizing influence on circadian rhythms. This suggests that the latter can be used as one of the reliable markers of accelerated ageing. Recent clinical studies depicted melatonin as a prophylactic and therapeutic remedy against neurological diseases such as Alzheimer and Parkinson diseases, Multiple sclerosis, Huntington disease, epilepsy, etc. [26].

In the studies concerned with this problem, much attention is paid to the role of peptides in preventing premature ageing. This approach is based on the existence of a bioregulating system in the human organism, which functions through cell mediators, oligopeptides whose main purpose is selective transmission of information during the interaction of the immune, nervous, endocrine and other systems [27].

When the organism is in a pathological state, the information transmission process is disrupted. As a result, a number of endogenic compounds are released and accumulated, including peptides transferring distorted information, which hinders coordinated operation of the regulating body systems. Consequently, introduction of physiologically reactive peptides into to the human body contributes to the restoration of self-regulation of organs and systems. Regulation of homeostasis through peptides takes an important place in the complicated chain of physiological processes leading to the ageing of cells, tissues, organs and the body as a whole [28]. Morphological and functional equivalent of ageing is involution of organs and tissues, and primarily those that are concerned with the main regulatory systems including the nervous, endocrine and immune ones. There are data available testifying to age hypoplasia, and in a number of cases, to the atrophy of the pineal gland, thymus, neuronal cortex and sub-cortex, retina, vascular wall and genitalia $[4,29$, 30].

For the first time in history, thanks to the development of a new concept based on application of a unique group of peptide geroprotectors, we have a realistic and affordable opportunity to prevent age-related diseases, by slowing down the ageing process and lengthening active life span. At present, with the existing level of knowledge, this is the only reliable way to affect the ageing process in humans [6].

Analysis of in vitro research results concerned with the effect of peptides allows making a general conclusion about the possibility of targeted induction of polypeptide cell differentiation, which is the basis for achieving longevity up to the species-specific limit. Using the DNA-microchip technology, a study was carried out concerned with the influence of a number of peptides on the expression of 15247 genes in the heart and brain of mice. In the experiments, clones were used from the DNA library of the National Institute on Aging, USA [31]. These experiments produced unique data on the expression of various genes under the peptide influence. An important conclusion was made about every peptide specifically regulating particular genes. The experimental results suggest the existence of a mechanism for peptides to regulate genetic activity.

Considering the data above, as well as in vivo experiments [9], which suggest high geroprotective reactivity of both natural tissue-specific and synthetic peptide preparations [32], research in the recent years was focused on studying the effectiveness of peptide preparations and peptides administered to old-aged people [33].

Thus, annual course of treatment with thymus and pineal gland preparations resulted in credible reduction of mortality in patients during the period observed (15 years). This was due to the improvement in the functions of the immune, endocrine, cardio-vascular systems, and the brain, as well as that of the bone tissue density.

Health preservation and prevention of premature ageing of the available work force will allow to withstand excessive demographic load in the context of global ageing tendency. The results of 35-year-long experimental tests and clinical research into the bioregulators developed at the Saint Petersburg Institute of Bioregulation and Gerontology by the staff of 
the leading Russian and foreign medical research institutions showed their high efficiency. Notably, administration of peptide bioregulators to over $15 \mathrm{mln}$ people demonstrated their complete safety because they actually constitute organism's metabolites.

The most important direction is preventive application of peptide bioregulators for improving resistance to the effect of destabilizing factors. This allows lowering the ageing rate, the risk of age-related pathology development and facilitates increasing life span, improving the life quality of older people.

The Concept of Healthy Ageing proposed by the UN experts as one of the priorities of the UN Research Agenda on Ageing for the $21^{\text {st }}$ century focuses on people's life style (dieting habits, abstaining from alcohol and smoking, etc.), which can have a marked positive effect on the rate of age-related diseases and, hence, on general life span. However, it should be emphasized that application of products normalizing age-related hormonal, metabolic and immunological changes and thus slowing down the genetic ageing process (by slowing down the ageing rate, rather than postponing its onset), is going to have a most significant geroprotective effect.

\section{References}

1. United Nations, (2002). Madrid International Plan of Action on Ageing. URL: https://www.un.org/development/desa/ageing

2. World Population Ageing 2017: Highlights. DESA, United Nations, New York, 2017.

3. V.Kh. Khavinson, O.N. Mikhailova, Health and Aging in Russia. In: Global Health and Global Aging, (Jossey-Bass, USA, AARP Foundation, 2007)

4. V. Khavinson, V. Morozov, Neuroendocrinol. Lett., 24, 233-40 (2003)

5. V.V. Frolkis, Aging and increased longevity, 237 (L., Nauka, 1988)

6. V.Kh. Khavinson, Neuroendocrinol. Lett. (2002)

7. I. Mechnikov, Etudes sur la nature humaine: essai de philosophie optimiste (Paris: Masson, 1903)

8. F. Jacob, J. Monod, J. Mol. Biol., 3, 318-56 (1961)

9. V. Khavinson, I. Popovich, Short Peptides Regulate Gene Expression, Protein Synthesis and Enhance Life Span. Ed. A.M. Vaiserman. Anti-aging Drugs: From Basic Research to Clinical Practice, 57, 496-513 (2017)

10. B.F. Vanyushin, V.Kh. Khavinson, Short Biologically Active Peptides as Epigenetic Modulators of Gene Activity. Eds. W. Doerfler, P. Böhm. Epigenetics - A Different Way of Looking at Genetics, 69-90 (2016)

11. L. Fontana, T.E. Meyer, S. Klein, J.O. Holloszy, Proc. Nat. Acad. Sci. USA, 101, 6659-63 (2004)

12. J.O. Holloszy, L. Fontana, Exp. Gerontol., 42, 709-12 (2007)

13. W.E. Kraus, M. Bhapkar, K.M. Huffman, et. al., Lancet Diabetes Endocrinol, 7, 67383 (2019) doi: 10.1016/S2213-8587(19)30151-2

14. D. Harman, J. Gerontol., 11, 298-300 (1956)

15. D. Harman, Ann. NY Acad. Sci., 717, 257-66 (1994)

16. B. Salehi, A.P. Mishra, M. Nigam, B. Sener, M. Kilic, M. Sharifi-Rad, P.V.T. Fokou, N. Martins, J. Sharifi-Rad, Biomedicines, 9(6(3)), 912018 doi: 10.3390/biomedicines6030091.

17. D.R. Valenzano, A. Cellerino, Cell Cycle, 10, 1027-32 (2006)

18. M. Jang, L. Cai, G.O. Udeani et al., Science, 275, 218-20 (1997)

19. P. Brasnyo, G.A. Molnar, M. Mohas et al., Br. J. Nutr., 106, 383-89 (2011)

20. J.L. Barger, T. Kayo, J.M. Vann et al., PloS One, 3, e2264 (2008)

21. S. Cho, K. Namkoong, M. Shin et al., J. Med. Food, 20, 323-34 (2017) doi: 10.1089/jmf.2016.3856. 
22. J.H. Chung, V. Manganiello, J.R. Dyck, Trends Cell. Biol., 22, 546-54 (2012)

23. W. Pierpaoli, W. Regelson, Proc. Natl. Acad. Sci.USA, 91, 787-91 (1994)

24. V.N. Anisimov, I.G. Popovich, M.A. Zabezhinski, S.V. Anisimov, G.M. Vesnushkin, I.A. Vinogradova, Biochim. Biophys. Acta, 1757, 573-89 (2006)

25. R.J. Reiter, D.X. Tan, Neuroendocrinol. Lett., 23, 3-8 (2002)

26. M. Gunata, H. Parlakpinar, H.A. Acet, Rev. Neurol. (Paris), 9, pii: S00353787(19)30882-3 (2019)

27. V.N. Anisimov, V.Kh. Khavinson, Small peptide-associated modulation of aging and longevity. Modulating aging and longevity. Ed. S.I.S. Rattan (Kluwer Academic Publishers (Printed in Great Britain), 2003)

28. V.Kh. Khavinson, Peptide regulation of ageing (SPb.: Humanistica, 2008)

29. V.N. Anisimov, V.Kh. Khavinson, V.G. Morozov, Ann. N.Y. Acad. Sci., 719, 483-93 (1994)

30. V.N. Anisimov, V.Kh. Khavinson, V.G. Morozov,The Gerontologist, 38, 7-8 (1998)

31. S.V. Anisimov, K.R. Bokheler, V.Kh. Khavinson, V.N. Anisimov, Bull. Exp. Biol. Med., 133(3), 293-9 (2002) doi: 10.1023/a:1015859322630.

32. V.Kh. Khavinson, V.V. Malinin, Gerontological aspects of genome peptide regulation (Basel (Switzerland): Karger AG, 2005)

33. O.V. Korkushko, V.Kh. Khavinson, V.B. Shatilo, I.A. Antonyk-Sheglova, Bull. Exp. Biol. Med., 151, 366-69 (2011) 